\title{
Intramural Pregnancy Misdiagnosed as Gestational Trophoblastic Neoplasia: Case Report and Review of the Literature
}

\author{
Lejing Zang and Yan $\mathrm{Hu}^{*}$
}

Department of Obstetrics and Gynecology, The First Affiliated Hospital of Wenzhou Medical University, Wenzhou, China

*Corresponding author: Yan Hu, Department of Obstetrics and Gynecology, The First Affiliated Hospital of Wenzhou Medical University, Wenzhou, 325000, China, Tel: +86-13806696807

\begin{abstract}
Purpose: To date, only a few cases of intramural pregnancy have been recorded. We aim to report an uncommon case of intramural pregnancy misdiagnosed as gestational trophoblastic neoplasia, emphasizing that earlier surgical intervention may contribute to timely diagnosis and precise treatment.

Methods: Relevant articles published over the past five years were identified through a literature searching, and related references in those literatures were also manually searched to find additional articles.

Results: Nineteen cases in total including this report were reviewed. Most patients possessed a history of intrauterine operation or uterine surgery. Diagnoses were mainly made by imageological examinations. And surgical procedure tended to be the dominant treatment of such diseases.

Conclusions: Intramural pregnancy is considered to be the rarest type of ectopic gestation, where the gestational sac is completely encircled by myometrium. The non-specific clinical manifestations and non-uniform imageological criteria lead to the difficult diagnoses. However, earlier surgical management may contribute to timely diagnosis and precise treatment.
\end{abstract}

\section{Keywords}

Gestational trophoblastic neoplasia, Intramural pregnancy, Management, Misdiagnosis

\section{Introduction}

Intramural pregnancy is an extremely rare form of ectopic pregnancy, and refers to extrauterine conception whose gestational sac (GS) is partially or completely encircled by the myometrium of the uterus, separating from the endometrial cavity, fallopian tubes, or round ligament. It accounts for less than $1 \%$ of the total number of ectopic pregnancies. Little is known about exact etiology of intramural pregnancy. Prior intrauterine trauma such as myomectomy, dilatation and curettage, cesarean section and difficult in vitro fertilization and embryo transfer (IVF-ET) may cause microscopic sinus tract and resultant improper implantation. Besides, the embryo will migrate into the myometrium on account of adenomyosis, together with endometrial tissue. Increased trophoblastic activity and inappropriate decidualization may also be predisposing factors [1-5]. The most common clinical manifestations of intramural pregnancy contain vaginal bleeding and abdominal pain, which are exactly unremarkable. Although early detection is possible owing to imageological technology being advanced, the condition remains unknown to many clinicians and sonographers.

We hereby report a case of intramural pregnancy that was misdiagnosed as gestational trophoblastic neoplasia (GTN). The patient gave informed consent for this presentation. In addition, we have made a comprehensive review of published cases on PubMed from January 2015 to November 2020 in this report, nearly 18 cases were found by using the keywords: Intramural pregnancy.

\section{Case Report}

A 33-year-old woman, gravida 4 para 1, presented

Citation: Zang L, Hu Y (2021) Intramural Pregnancy Misdiagnosed as Gestational Trophoblastic Neoplasia: Case Report and Review of the Literature. Obstet Gynecol Cases Rev 8:213. doi. org/10.23937/2377-9004/1410213

Accepted: November 11, 2021: Published: November 13, 2021

Copyright: (C) 2021 Zang L, et al. This is an open-access article distributed under the terms of the Creative Commons Attribution License, which permits unrestricted use, distribution, and reproduction in any medium, provided the original author and source are credited. 


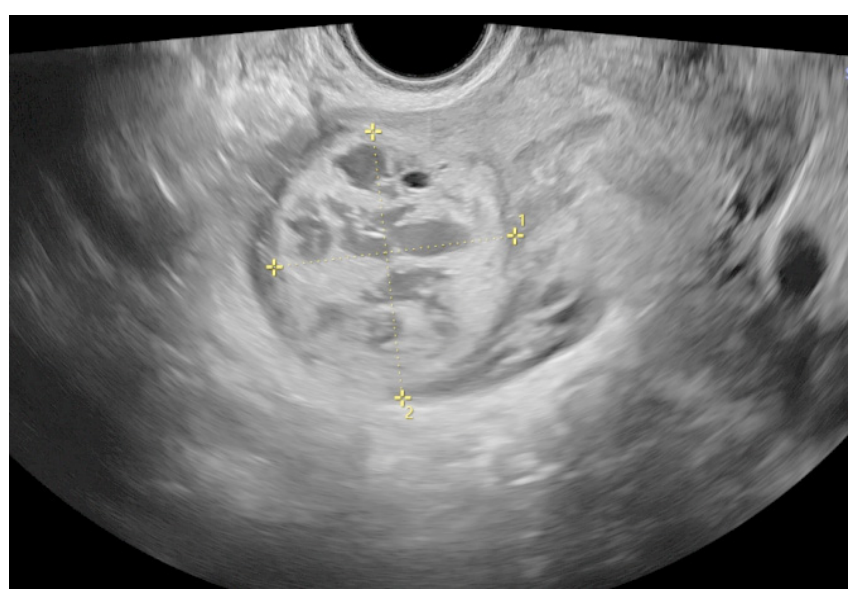

A

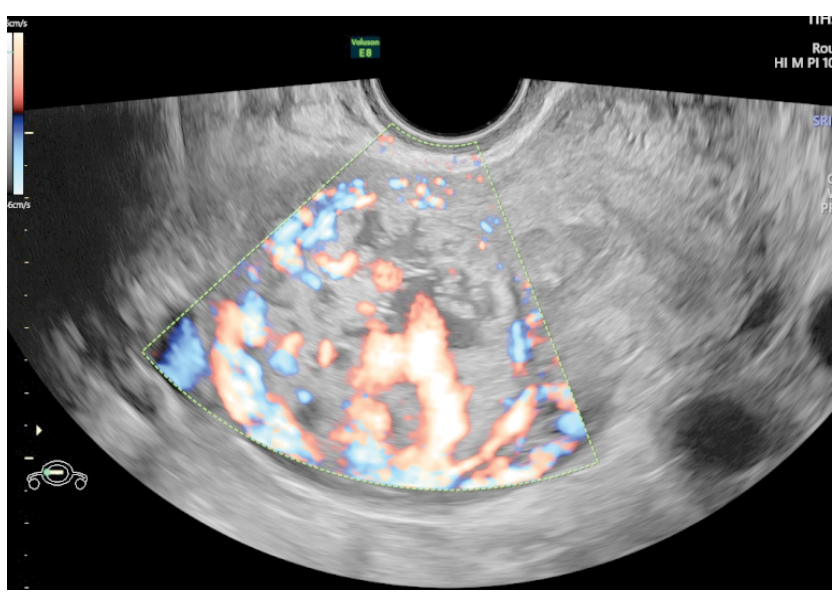

B

Figure 1: (a) Transvaginal scan showed a mass with uneven echo in the right lateral wall of the uterus; (b) Doppler flow revealed abundant blood flow signals.

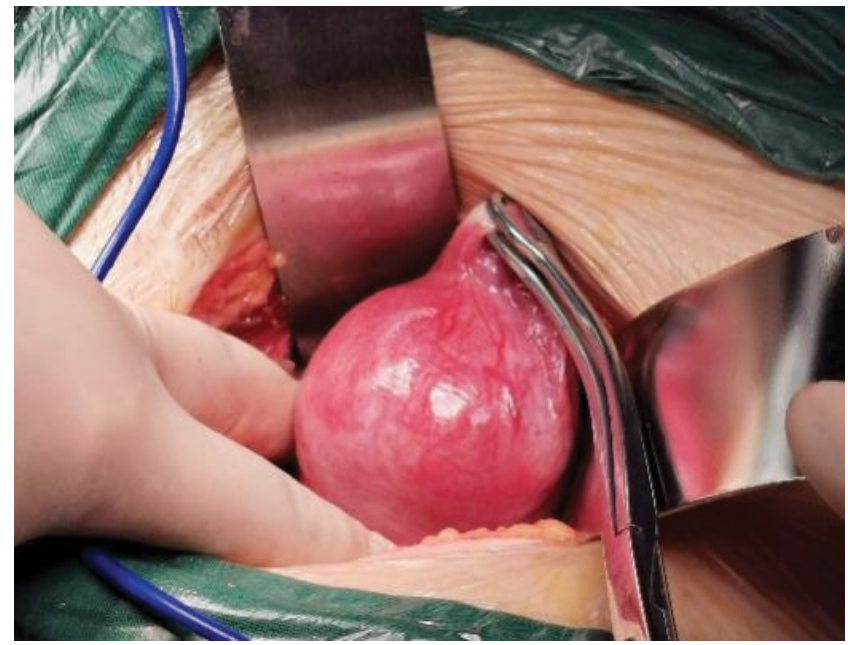

A

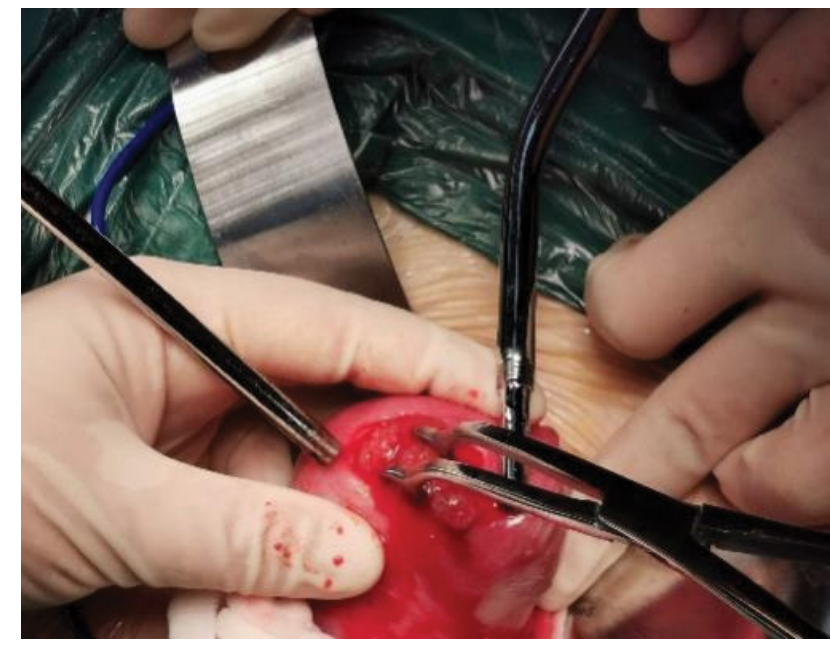

B

Figure 2: (a) Laparoscopic intra-operative view showed the gestational sac protruding out from below the right horn of the uterus; (b) The chorionic villous tissues bulged out after a fusiform incision being made.

with vaginal bleeding, slight chest tightness and nausea, underwent a curettage at 5 weeks' gestation for incomplete abortion. While the level of serum $\beta$-HCG showed $39576 \mathrm{IU} / \mathrm{L}$ on post-surgery day and continued to rise during the following 2 weeks. On the day being transferred to our hospital, the level of serum $\beta$-HCG manifested $168208 \mathrm{IU} / \mathrm{L}$, and the transvaginal sonography (TVS) denoted a mass with uneven echo and abundant blood flow in the right lateral wall of uterus, measuring $38 \times 43 \times 40 \mathrm{~mm}$ (Figure 1 a and Figure $1 \mathrm{~b}$ ). On the second day of admission, the $\beta$-hCG value rose to $216338 \mathrm{IU} / \mathrm{L}$, along with the mass growing to $41 \times$ $44 \times 52 \mathrm{~mm}$. However, the chest computed tomography (CT) and craniocerebral CT revealed no abnormalities. Combining with the pathological result of her previous curettage which indicated necrotic villi, decidual tissue and some trophoblast cells, the preliminary diagnosis of
GTN and the decision to initiate EMA/CO chemotherapy regimen were made. Two days later, the serum $\beta$-HCG ascended to $225000 \mathrm{IU} / \mathrm{L}$, and the ultrasound did not see the reduction of the mass. Due to the unsatisfactory effect, a surgical method was adopted on the tenth day. During the hysteroscopy exploration, a projection surrounded by abundant and tortuous vascular vessels was seen. Rapid pathology manifested the endometrium clamped by forceps did not contain any abnormal composition, leading to the exploratory laparotomy which showed a bulging mass measuring 50 $\times 50 \mathrm{~mm}$ with a purplish-blue-colored surface below the right horn of the uterus (Figure 2a). The chorionic villous tissues bulged out after a fusiform incision was made by a coagulation hook (Figure $2 b$ ), then the product of conception was completely removed and the wound was continuously sutured. Intraoperative pathology showed 


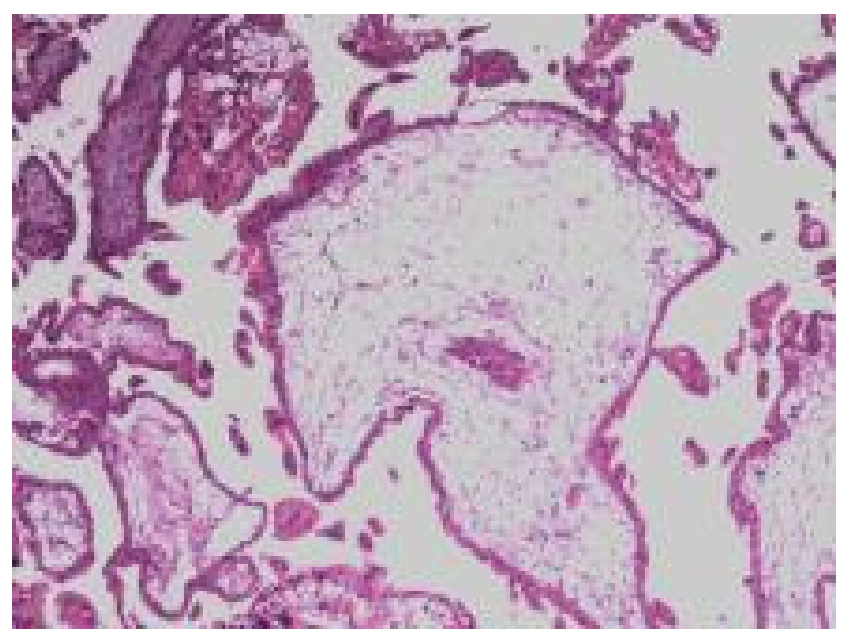

A

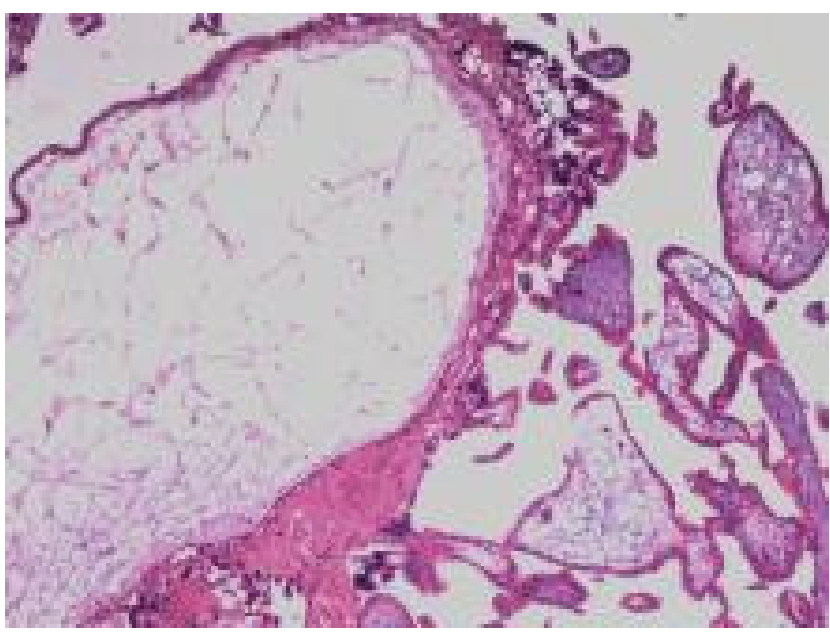

B

Figure 3: $(a$ and b) Histopathological observation showed the characteristic chorionic villi and the smooth muscle tissue was infiltrated with trophoblast cells.

oedematous villi and proliferous trophoblast cells, so the diagnosis of the hydatidiform mole was considered. Given such consequence, adjuvant chemotherapy continued after surgery. One day postoperatively the patient's $\beta$-hCG titer dropped to $37955 \mathrm{IU} / \mathrm{L}$ and then decreased to $711 \mathrm{IU} / \mathrm{L}$ on the tenth day, at which point she was discharged. A follow-up demonstrated the $\beta$ hCG level returned to normal. Ultimately, postoperative pathology in combination with immunohistochemistry analysis (P57+) confirmed chorionic villi surrounded by myometrium with no identifiable fallopian tube, validating the diagnosis of intramural ectopic pregnancy (Figure $3 a$ and Figure $3 b$ ).

\section{Discussion}

As is considered to be the rarest ectopic gestation, the incidence of intramural pregnancy is about 1:30000. Owing to its low incidence, it is easily misdiagnosed. According to Kirk, et al. [6], only 22 cases of intramural pregnancy from 2000 to 2012 all over the world. Our report summaries the literature of intramural pregnancies since the year 2015, revealing 18 cases in all, the details of them are summed up in Table 1 . There was only one patient led to a live birth [7], nevertheless, 10 patients preserved the fertility successfully.

The pathogenesis of intramural pregnancy still keeps unclear, various clinical conditions mirroring erstwhile traumas have been reported: Pelvic inflammation; endometrial defects; tiny sinus in the myometrium as a result of the previous operation; and pregnancy with adenomyosis. In our review, the age of patients ranged from 19 to 41-years-old. Except for seven patients, approximately three quarters $(77.8 \%)$ had a history of uterine trauma [1-5,7-11], including artificial abortion (33.3\%), cesarean section $(21.4 \%)$ and myomectomy (14.3\%). One cases (5.6\%) experienced intrauterine device (IUD) placement, and two (11.1\%) were the result of adenomyosis or endometriosis. Furthermore, two patients (11.1\%) became gravid with the aid of IVF-ET $[7,11]$. Similarly, the patient in our case had undergone several times of uterine curettage.

Vaginal bleeding (27.8\%) and abdominal pain (16.7\%) were the two relative common clinical manifestations in this summary, and our patient suffered from both of them. Such symptoms are unrepresentative so that late diagnosis or misdiagnosis could be made. Recently, advanced imageological examinations may matter by identifying trophoblastic invasion which extends beyond the endometrial-myometrial junction into the myometrium. Transvaginal ultrasound (TVS) and magnetic resonance imaging (MRI) are common auxiliary techniques being performed, of which the latter is widely considered to be the gold standard $[3,7]$. Memtsa, et al. [12] even proposed a series of criteria for the sonographic diagnosis of intramural pregnancy. Apart from one case (7.1\%) lacked related information, almost all patients (92.9\%) underwent ultrasound examinations, some of whom accepted additional MRIs. Our case utilized both aforementioned auxiliary modalities, still more, added CT was performed for exclusion of choriocarcinoma which may metastasize to other organs. Regretablly, combining with the extremely high level of serum $\beta-h C G$, the diagnosis of GTN was primarily made.

Treatments of intramural pregnancy vary based on the exact location of the GS, depth of muscular invasion, patient's condition and desire to conserve fertility. If the uterus ruptures, hysterectomy is inevitable for the massive hemorrhage. If a clear diagnosis and hemodynamic stability can be achieved, conservative management could be a better choice. Injection of methotrexate (MTX) locally or systemically is the most convenient alternative, and studies have reported that ultrasound-guided intra-sac injection (USGI) of MTX is 


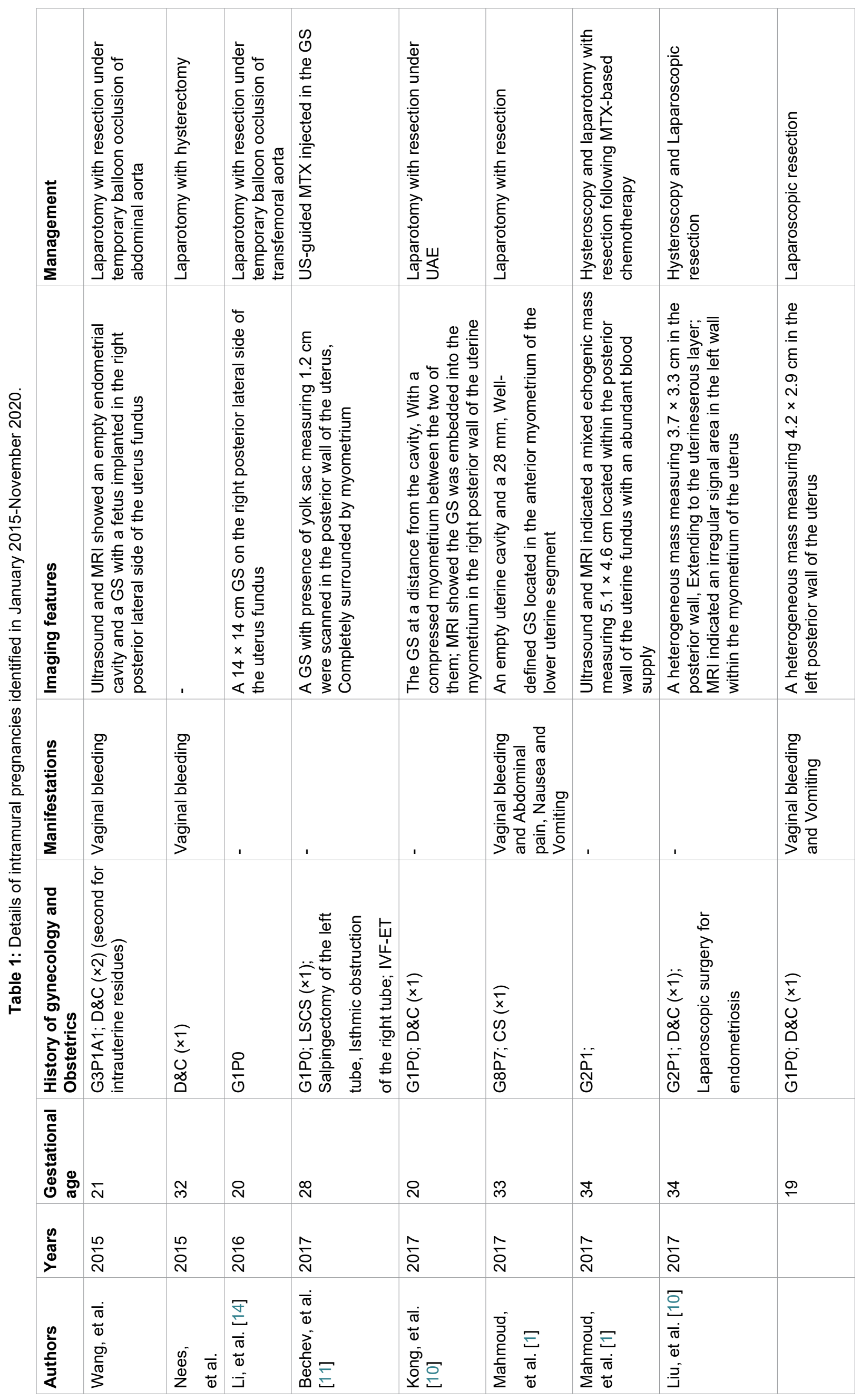




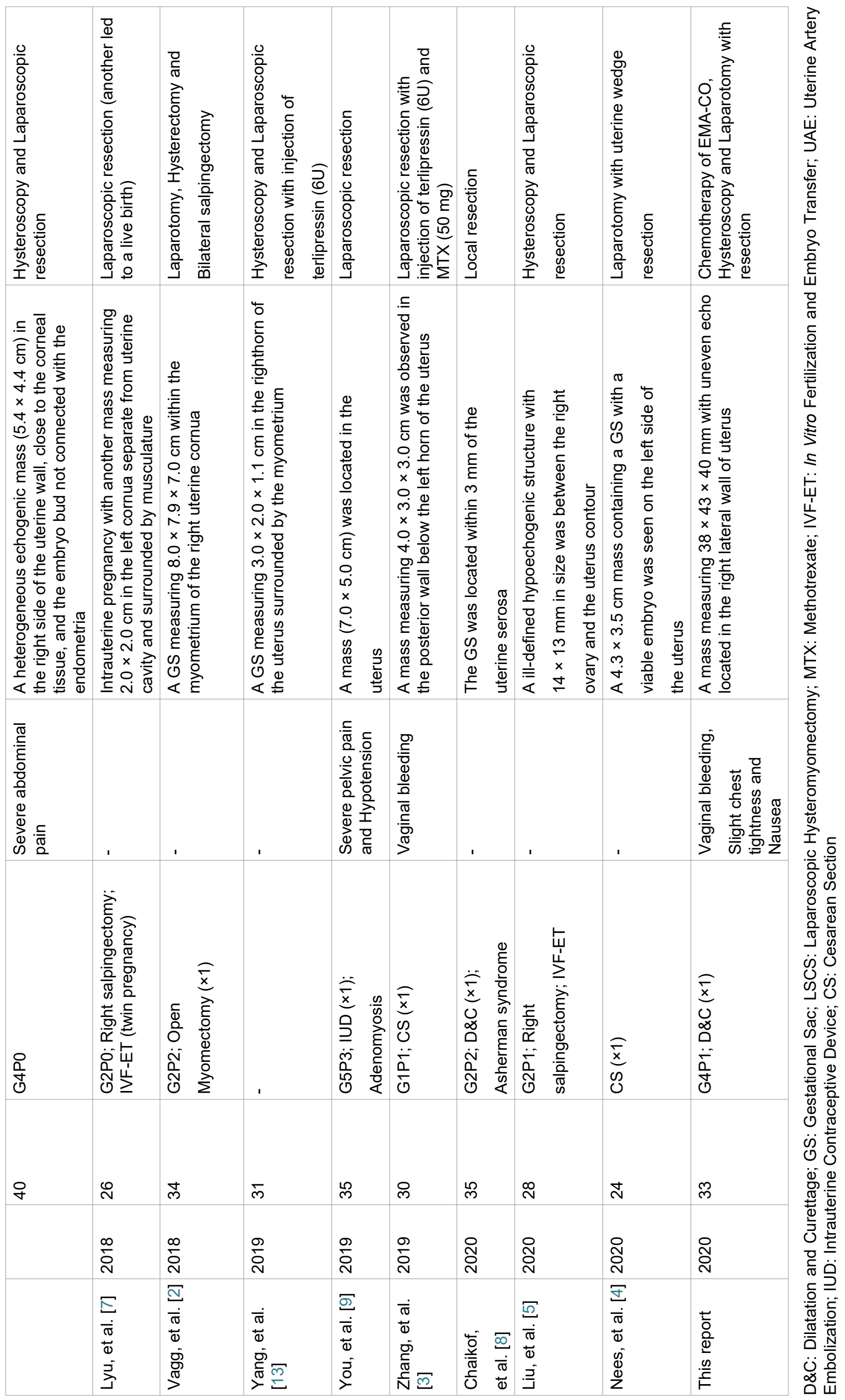


evaluated relatively more effective unless the $\beta$-HCG indicates an extraordinarily low concentration [11]. Local removal under laparoscope is always accepted when the GS can be observed through real-time exploration. Yang, et al. [13] suggested hysteroscopic resection is feasible as well, which depends on the distance between GS to uterine cavity or uterine serosa. If there exists uncertainty in minimally invasive surgery, an open laparotomy could be another option. Nevertheless, surgical strategy theoretically increases the danger of uterine rupture in the event of a future pregnancy. Furthermore, uterine artery embolization (UAE) and temporary balloon occlusion have been confirmed effective in embryo atrophy and absorption as well [14].

The majority of cases (55.6\%) we reviewed accepted minimally invasive surgery with local resection, indicating the effectiveness and practicability in where advanced endoscopic expertise is available. One (7.1\%) was amenable to USGI of MTX, and one (7.1\%) was treated by expectant management for the desperately low level of serum $\beta$-hCG. In our report, EMA/CO chemotherapy regimen was adopted originally for suspect of GTN, a kind of malignant gynecological tumor caused by trophoblastic proliferation, yet the $\beta$-hCG level of which could far exceed that of intramural pregnancy. If the treatment cannot be distinctly decided, the surgical procedure should be considered, with which unnecessary chemotherapy could be avoided. Sun, et al. [7] described a similar case to the one here, owing to misdiagnosis and the implementation of chemotherapy, the patient was exposed to a series of side effects, including fever, fatigue, mouth ulcers, liver dysfunction and bone marrow suppression. Fortunately, our patient has promptly accepted surgery, confirming the diagnosis of intramural pregnancy and curing it ultimately.

\section{Conclusion}

This case and review of the literature emphasize the challenge of recognizing intramural pregnancy. Early diagnosis plays a pivotal role in preventing complications, including uterine rupture, lifethreatening hemorrhage and resultant hysterectomy. Atypical clinical presentations and indistinguishable imaging features are primary difficulties for medical professionals. The treatment should be individualized and combined, yet if intramural pregnancy is suspected, earlier surgical intervention may contribute to timely diagnosis and supervision. In addition, patients exposed to hazardous factors ought to seek medical attention early in their pregnancy. It is also vital to set up uniform diagnostic criteria and management guidelines.

\section{Disclosure}

The authors declare no conflict of interest.

\section{Author Contribution}

Lejing Zang: Project development, Data Collection, Manuscript writing; Yan Hu: Supervision.

\section{References}

1. Mahmoud OA, Mahmoud MZ (2017) A rare case of ectopic pregnancy in a caesarean section scar: A case report. BJR Case Rep 3: 20170010.

2. Vagg D, Arsala L, Kathurusinghe S, Ang WC (2018) Intramural ectopic pregnancy following myomectomy. J Investig Med High Impact Case Rep 6.

3. Zhang Q, Xing X, Liu S, Xie X, Liu X, et al. (2019) Intramural ectopic pregnancy following pelvic adhesion: Case report and literature review. Arch Gynecol Obstet 300: 1507-1520.

4. Nees J, Faigle-Krehl G, Brucker J, Leucht D (2020) Intramural pregnancy: A case report. Case Rep Womens Health 27: e00215.

5. Liu $\mathrm{Y}, \mathrm{Wu} Y$ (2020) Intramyometrial pregnancy after cryopreserved embryo transfer: A case report. BMC Pregnancy Childbirth 20: 90.

6. Kirk E, McDonald K, Rees J, Govind A (2013) Intramural ectopic pregnancy: A case and review of the literature. Eur J Obstet Gynecol Reprod Biol 168: 129-133.

7. Lyu J, Sun W, Lin Y (2018) Successful management of heterotopic intramural pregnancy leading to a live birth of the intrauterine pregnancy. J Minim Invasive Gynecol 25: 1126-1127.

8. Chaikof M, Hobson S, Sobel M (2020) Fundal intramural ectopic pregnancy. CMAJ 192: E1211.

9. You SH, Chen PC, Chueh HY, Huang KG, Wang, HS, et al. (2019) Perforating scar of intrauterine device with subsequent intramural pregnancy and uterine rupture. J Minim Invasive Gynecol 26: 591-592.

10. Liu NN, Han XS, Guo XJ, Sun LT, Kong XC (2017) Ultrasound diagnosis of intramural pregnancy. J Obstet Gynecol Res 43: 1071-1075.

11. Bechev B, Konovalova M (2017) A very rare case of ectopic intramural pregnancy after IVF-ET. Clin Exp Obstet Gynecol 44: 802-803.

12. Memtsa $M$, Jamil $A$, Sebire $N$, Jauniaux $E$, Jurkovic $D$ (2013) Diagnosis and management of intramural ectopic pregnancy. Ultrasound Obstet Gynecol 42: 359-362.

13. Yang B, Zhu D, Tang J, Ran S, Li L, et al. (2020) Management of intramural pregnancy by hysteroscopic surgery. J Minim Invasive Gynecol 27: 995-996.

14. Li S, Liu H, Li X, Liu Z, Li Y, et al. (2016) Transfemoral temporary aortic balloon occlusion in surgical treatment of second trimester intramural ectopic pregnancy. J Obstet Gynaecol Res 42: 716-718. 